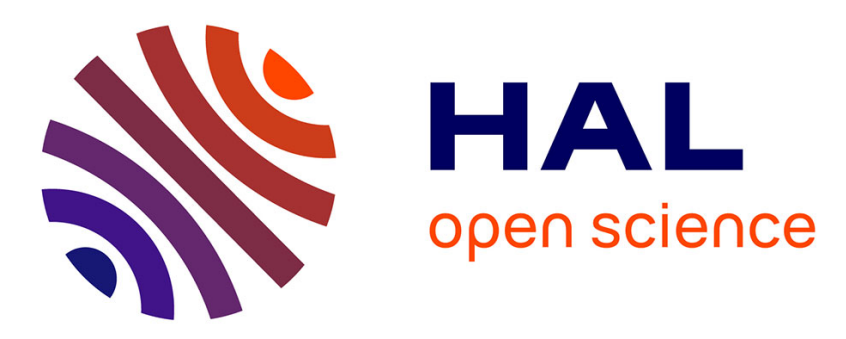

\title{
Uniform stability of sets for difference inclusions under summability criteria
}

\author{
Andrew Teel, Dragan Nesic, Antonio Loria, Elena Panteley
}

\section{To cite this version:}

Andrew Teel, Dragan Nesic, Antonio Loria, Elena Panteley. Uniform stability of sets for difference inclusions under summability criteria. CDC 2009, Dec 2009, Shanghai, China. pp.4131-4136, 10.1109/CDC.2009.5399608 . hal-00831373

\section{HAL Id: hal-00831373}

https://hal-centralesupelec.archives-ouvertes.fr/hal-00831373

Submitted on 6 Jun 2013

HAL is a multi-disciplinary open access archive for the deposit and dissemination of scientific research documents, whether they are published or not. The documents may come from teaching and research institutions in France or abroad, or from public or private research centers.
L'archive ouverte pluridisciplinaire HAL, est destinée au dépôt et à la diffusion de documents scientifiques de niveau recherche, publiés ou non, émanant des établissements d'enseignement et de recherche français ou étrangers, des laboratoires publics ou privés. 


\section{Uniform stability of sets for difference inclusions under summability criteria}

\author{
Andrew R. Teel \\ Dragan Nešić
}

\begin{abstract}
We present equivalent characterizations of uniform global exponential stability and uniform global asymptotic stability of arbitrary closed not necessarily compact sets for nonlinear difference inclusions. Our conditions are established in the form of summability criteria that do not require the knowledge of a Lyapunov function.
\end{abstract}

\section{INTRODUCTION}

We study discrete-time systems with discontinuous righthand sides, more precisely, systems described by difference inclusions

$$
x^{+} \in F(x),
$$

where $x \in \mathbb{R}^{n}$ and $F(\cdot)$ is in general a set-valued map (more specific conditions on it will be given later).

The study of such class of systems is important for a number of reasons: for example, they appear in the search for periodic solutions of continuous-time non-autonomous systems by defining a Poincaré map - $c f$. [2]. Also, analysis of discrete-time systems may appear as an intermediary step in the study of sampled-data systems $-c f$. [12] via approximately discretized models $-c f$. [4]. In that respect, it is generally accepted that discretized systems remain 'stable' under small time-step discretization. However, this entails specificities which must be studied in their own right such as the characterization of attractors, from a numerics viewpoint $-c f$. [7] and the introduction of appropriate definitions of stability for discrete-time systems $-c f$. [11].

Analysis methods alternative to Lyapunov's, which remains the most developed $-c f$. [6], [3], [17], are so-called integrability criteria $-c f$. [15] for continuous-time systems. These are stated as conditions on functions of the trajectories that have to be (uniformly) integrable i.e., the integral of a function of time over $\mathbb{R}_{+}$with certain monotonicity properties, along the trajectories of the system, must be bounded from above by a quantity that does not depend on initial times. Integrability criteria are useful in situations when the uniform stability has already been established (for instance, via a positive definite function whose derivative along the solutions of the system is negative semi-definite) and one only needs to check the uniform attractivity. Integrability conditions establish a clear link with inputoutput stability $-c f$. [8] in the classical $\mathcal{L}_{p}$ sense and allow

A. R. Teel is with Electrical and Computer Engineering Department, The University of California, Santa Barbara, CA 93106-9560, USA; D. Nešić is with the Department of Electrical and Electronic Engineering, The University of Melbourne, Parkville, 3010, Victoria, Australia, d.nesiclee.unimelb.edu.au; A. Loría and E. panteley are with with C.N.R.S. at Laboratoire des Signaux et Systèmes, SUPELEC, 3, Rue Joliot Curie, 91192 Gif s/Yvette, France. loria@lss.supelec.fr, panteleyelss.supelec.fr

\author{
Antonio Loría Elena Panteley
}

us to establish convergence rates for dynamical systems. Closer to the realm of engineering science integrability conditions may result useful in applied control design; for instance in cases where Lyapunov-like methods fail.

In the discrete-time context integrability conditions take the form of summability criteria and may also be considered as conditions on convergence of infinite series. Of broader interest are necessary and sufficient conditions for stability of sets. A sampled-data counterpart of the integrability criteria for differential inclusions from [15] was reported in [13]. In this approach, one needs to establish stability of a family of approximate discrete-time models that are parameterized with the sampling period and the goal is to establish appropriate stability properties of this family that would guarantee that the family of exact discrete-time models will also be stable for sufficiently small sampling periods.

Results in [13] can be modified and used in the special case when the exact discrete time model of the sampleddata system is known and one does not need to deal with families of discrete-time systems. However, straightforward modifications of results from [13] to this special case would be unnecessarily restrictive and technical. Hence, in this paper we address the case when the exact discretetime model of the system is known and we prove the results under weaker assumptions than what would be possible by doing the modifications of [13]. In particular, we investigate various stability characterization of uniform global asymptotic (and exponential) stability of arbitrary sets for nonlinear difference inclusions (I). We emphasize that very little is assumed on the set-valued map $F(\cdot)$ and, in particular, we allow $F(\cdot)$ to be discontinuous. Hence, our results hold under very general assumptions and, moreover, the proofs are less technical than their continuous-time or sampled-data counterparts. The results presented in this paper parallel the integral characterizations of stability of arbitrary sets for continuous time nonlinear differential inclusions $-c f$. [15] and constitute an outgrowth of the main results in [8] for difference equations.

\section{PRELIMinaries}

$\mathbb{R}$ and $\mathbb{N}$ denote, respectively, the sets of real and natural (that includes zero) numbers. Given $c \in \mathbb{R}$ we denote as $\mathbb{R}_{\geq c}$ the set of all real numbers that are greater than or equal to $c$ (similar notation is used for the set $\mathbb{N}$ ). Given a closed set $\mathcal{A} \subset \mathbb{R}^{n}$, we denote the distance of an arbitrary $x \in \mathbb{R}^{n}$ from this set as:

$$
|x|_{\mathcal{A}}:=\inf _{z \in \mathcal{A}}|x-z| .
$$


Also, given $0 \leq \delta \leq \Delta$, we use the notation $\mathcal{H}_{\mathcal{A}}(\delta, \Delta):=$ $\left\{x \in \mathbb{R}^{n}: \delta \leq|x|_{\mathcal{A}} \leq \Delta\right\}$.

Assumption 1 Consider system (1). We assume that for each $x$ the set $F(x)$ is non-empty.

The solutions with initial condition $x_{0} \in \mathbb{R}^{n}$ are denoted by $\phi\left(\cdot, x_{0}\right)$ hence, $\phi\left(0, x_{0}\right)=x_{0}$. When $F(x)$ is multivalued, the solution generated by the initial condition $x_{0}$ is not uniquely defined. We denote the set of all possible solutions starting from $x_{0}$ as $\mathcal{S}\left(x_{0}\right)$ and for any function $\left(k, x_{0}\right) \mapsto \phi\left(k, x_{0}\right)$ we write $\phi \in \mathcal{S}\left(x_{0}\right)$ if we have that $\phi\left(k+1, x_{0}\right) \in F\left(\phi\left(k, x_{0}\right)\right)$ for all $k \in \mathbb{N}$.

We also use the following standard definitions. A function $\alpha: \mathbb{R}_{\geq 0} \rightarrow \mathbb{R}_{\geq 0}$ is said to belong to class $\mathcal{P D}$ (also $\alpha \in \mathcal{P} \mathcal{D}$ ) if it is continuous, zero at zero and positive for all other values of its argument. A function $\alpha: \mathbb{R}_{>0} \rightarrow \mathbb{R}_{>0}$ is said to belong to class- $\mathcal{G}$ (also $\alpha \in \mathcal{G}$ ) if the function is continuous, zero at zero and nondecreasing. It is said to belong to class- $\mathcal{K}$ (also $\alpha \in \mathcal{K}$ ) if $\alpha \in \mathcal{G}$ and it is strictly increasing. It is said to belong to class- $\mathcal{K}_{\infty}$ (also $\alpha \in \mathcal{K}_{\infty}$ ) if $\alpha \in \mathcal{K}$ and it is unbounded. Note that class- $\mathcal{K}_{\infty}$ functions are globally invertible. A function $\beta: \mathbb{R}_{\geq 0} \times \mathbb{N}_{\geq 0} \rightarrow \mathbb{R}_{\geq 0}$ is said to belong to class- $\mathcal{K} \mathcal{L}$ (also $\bar{\beta} \in \mathcal{K} \mathcal{L}$ ) if the function is nondecreasing is its first argument, non-increasing in its second argument and $\lim _{s \rightarrow 0^{+}} \beta(s, k)=\lim _{k \rightarrow \infty} \beta(s, k)=0$. The following lemma can be proved in a similar manner as the "Sontag's lemma" given in [14]:

Lemma 1 Let $\beta \in \mathcal{K} \mathcal{L}$ and $\lambda \in(0,1)$. There exist $\alpha_{1}, \alpha_{2} \in \mathcal{K}_{\infty}$ such that

$$
\alpha_{1}(\beta(s, k)) \leq \alpha_{2}(s) \lambda^{k} \quad \forall s, k .
$$

The following definition is not standard but we find it useful to state our main results.

Definition 1 A pair of class-G functions $\left(\alpha_{1}, \alpha_{2}\right)$ is said to be compatible for uniform global exponential stability (cUGES) if there exist $\underline{\lambda}, \bar{\lambda} \in(0,1)$ and $\bar{\Gamma} \geq 1$ such that

$\alpha_{2}(s) \leq \alpha_{1}(\bar{\Gamma} \cdot s) ; \quad \underline{\lambda} \cdot \alpha_{2}(s) \leq \alpha_{2}(\bar{\lambda} \cdot s) \quad \forall s \geq 0$.

For instance, functions $\alpha_{i}(s)=a_{i} s^{p}, i=1,2$ are cUGES if $a_{i}>0, i=1,2$ and $p>0$. Similarly, the functions $\alpha_{1}(s)=\alpha_{2}(s)=\arctan (s)$ are cUGES (for instance, we can take $\bar{\Gamma}=1, \underline{\lambda}=\bar{\lambda}=0.5)$.

Lemma 2 If the pair of class-G functions $\left(\alpha_{1}, \alpha_{2}\right)$ is cUGES, then for each $\mu \in[0,1)$ there exist $\Gamma \in \mathbb{R}_{\geq 1}$ and $\lambda \in[0,1)$ such that:

$$
\mu^{k} \alpha_{2}(s) \leq \alpha_{1}\left(s \cdot \Gamma \lambda^{k}\right) \quad \forall s \in \mathbb{R}_{\geq 0}, k \in \mathbb{N} .
$$

Remark 1 It is straightforward to show that if the pair $\left(\alpha_{1}(s), \alpha_{2}(s)\right)$ is cUGES, then for any $\Gamma \in \mathbb{R}_{\geq 1}$ we have that the pair $\left(\alpha_{1}(s), \alpha_{2}(\Gamma \cdot s)\right)$ is cUGES.

\section{CharaCterizations OF STABILITY OF SETS}

\section{A. Uniform global exponential stability of sets}

Let $\mathcal{A} \subset \mathbb{R}^{n}$ be a (given) closed (not necessarily compact) set. We introduce the following definitions for system (1).

Definition $2 \mathcal{A} \subset \mathbb{R}^{n}$ is uniformly globally exponentially stable (UGES) for system (1) if there exist $\Gamma \in \mathbb{R}_{\geq 1}$ and $\lambda \in[0,1)$ such that for all $x_{0} \in \mathbb{R}^{n}$ and all $\phi \in \mathcal{S}\left(x_{0}\right)$ we have

$$
\left|\phi\left(k, x_{0}\right)\right|_{\mathcal{A}} \leq \Gamma \lambda^{k}\left|x_{0}\right|_{\mathcal{A}} \quad \forall k \in \mathbb{N} .
$$

When $\mathcal{A}=\{0\}$ UGES boils down to the usual definition of uniform exponential stability of the origin.

Definition 3 The closed set $\mathcal{A}$ is finite-step contractive (FSC) for system (1) if there exist $k^{*} \in \mathbb{N}$ and $\lambda_{\circ} \in[0,1)$ such that for each $x_{0} \in \mathbb{R}^{n}$ and each $\phi \in \mathcal{S}\left(x_{0}\right)$ there exists $k \in\left\{0, \ldots, k^{*}\right\}$ such that

$$
\left|\phi\left(k, x_{0}\right)\right|_{\mathcal{A}} \leq \lambda_{\circ} \cdot\left|x_{0}\right|_{\mathcal{A}} .
$$

The following result establishes several equivalent characterizations of UGES for system (1).

Theorem 1 The statements enumerated below are equivalent:

1) $\mathcal{A} \subset \mathbb{R}^{n}$ is UGES for system (1);

2) $\mathcal{A} \subset \mathbb{R}^{n}$ is FSC for system (1) and there exists $\Gamma_{1} \in$ $\mathbb{R}_{\geq 1}$ such that

$$
|w|_{\mathcal{A}} \leq|x|_{\mathcal{A}} \cdot \Gamma_{1} \quad \forall x \in \mathbb{R}^{n}, w \in F(x) ;
$$

3) there exist $\alpha_{1} \in \mathcal{K}_{\infty}, \alpha_{2} \in \mathcal{G}$ such that the pair $\left(\alpha_{1}, \alpha_{2}\right)$ is cUGES and for each $x_{0} \in \mathbb{R}^{n}$ and each $\phi \in \mathcal{S}\left(x_{0}\right)$ we have

$$
\sum_{k=0}^{\infty} \alpha_{1}\left(\left|\phi\left(k, x_{0}\right)\right|_{\mathcal{A}}\right) \leq \alpha_{2}\left(\left|x_{0}\right|_{\mathcal{A}}\right) ;
$$

4) for each $\alpha_{1}, \alpha_{2} \in \mathcal{K}_{\infty}$ such that $\left(\alpha_{1}^{-1}, \bar{\Gamma} \cdot \alpha_{2}^{-1}\right)$ is cUGES for all $\bar{\Gamma} \in \mathbb{R}_{\geq 1}$, there exists $\Gamma_{2} \in \mathbb{R}_{\geq 1}$ such that for each $x_{0} \in \mathbb{R}^{n}$ and $\phi \in \mathcal{S}\left(x_{0}\right)$ we have

$$
\sum_{k=0}^{\infty} \alpha_{1}\left(\left|\phi\left(k, x_{0}\right)\right|_{\mathcal{A}}\right) \leq \Gamma_{2} \cdot \alpha_{2}\left(\left|x_{0}\right|_{\mathcal{A}}\right) .
$$

Remark 2 A continuous-time counterpart of Theorem 1 is given in [15, Theorem 2]. A sampled-data counterpart of this results is given in [13]. We note that the notion of cUGES was not used in [15], [13].

\section{B. Uniform global asymptotic stability of sets}

For closed sets $\mathcal{A} \subset \mathbb{R}^{n}$ and solutions $\phi \in \mathcal{S}\left(x_{0}\right)$ of systems (1) we introduce the more general definitions of asymptotic stability. 
Definition 4 The closed set $\mathcal{A} \subset \mathbb{R}^{n}$ is uniformly stable if for each $\epsilon>0$ there exists $\delta(\epsilon)>0$ such that for all $x_{0} \in \mathbb{R}^{n}$ satisfying $\left|x_{0}\right|_{\mathcal{A}} \leq \delta$, we have $\left|\phi\left(k, x_{0}\right)\right|_{\mathcal{A}} \leq \epsilon$ for all $k \in \mathbb{N}$ and all $\phi \in \mathcal{S}\left(x_{0}\right)$. The set $\mathcal{A}$ is uniformly globally stable (UGS) if moreover $\delta$ has the property that $\delta \rightarrow \infty$ as $\epsilon \rightarrow \infty$.

Remark 3 Following [5], we can show that the set $\mathcal{A}$ is UGS if and only if there exists $\rho \in \mathcal{K}_{\infty}$ such that for all $x_{0} \in \mathbb{R}^{n}$ and $\phi \in \mathcal{S}\left(x_{0}\right)$ we have

$$
\left|\phi\left(k, x_{0}\right)\right|_{\mathcal{A}} \leq \rho\left(\left|x_{0}\right|_{\mathcal{A}}\right) \quad \forall k \in \mathbb{N} .
$$

Moreover, if $\rho$ is a linear function we say that the set $\mathcal{A}$ is UGS (for system (1)) with linear growth.

Definition 5 The closed set $\mathcal{A} \subset \mathbb{R}^{n}$ is uniformly globally attractive (UGA) for system (1) if for each pair of strictly positive reals $(r, \epsilon)$ there exists $k^{*} \in \mathbb{N}$ such that:

$$
\left|x_{0}\right|_{\mathcal{A}} \leq r, \phi \in \mathcal{S}\left(x_{0}\right), \quad k \geq k^{*} \Longrightarrow\left|\phi\left(k, x_{0}\right)\right|_{\mathcal{A}} \leq \epsilon .
$$

Remark 4 Similarly to UGS we have the following characterization of UGAS. The set $\mathcal{A}$ is UGAS if there exists $\beta \in \mathcal{K} \mathcal{L}$ such that for all $x_{0} \in \mathbb{R}^{n}$ and all $\phi \in \mathcal{S}\left(x_{0}\right)$ we have

$$
\left|\phi\left(k, x_{0}\right)\right|_{\mathcal{A}} \leq \beta\left(\left|x_{0}\right|_{\mathcal{A}}, k\right) \quad \forall k \in \mathbb{N} .
$$

When $\mathcal{A}=\{0\}$ we recover Barbashin's definition of UGAS (of the null solution) -cf. [1].

The following result establishes several equivalent characterizations of UGAS of sets for system (1).

Theorem 2 The following statements are equivalent:

1) the closed set $\mathcal{A}$ is UGAS for system (1);

2) a) the closed set $\mathcal{A}$ is UGA for system (1) and

b) there exists $\rho \in \mathcal{K}_{\infty}$ such that for all $x \in \mathbb{R}^{n}$ and all $w \in F(x)$ we have $|w|_{\mathcal{A}} \leq \rho\left(|x|_{\mathcal{A}}\right)$;

3) a) $\mathcal{A}$ is UGS for system (1) and

b) there exists $\eta \in \mathcal{P D}$ and $\alpha_{2} \in \mathcal{G}$ such that for all $x_{0} \in \mathbb{R}^{n}$ and all $\phi \in \mathcal{S}\left(x_{0}\right)$ we have

$$
\sum_{k=0}^{\infty} \eta\left(\left|\phi\left(k, x_{0}\right)\right|_{\mathcal{A}}\right) \leq \alpha_{2}\left(\left|x_{0}\right|_{\mathcal{A}}\right)
$$

4) a) there exists $\rho \in \mathcal{K}_{\infty}$ such that for all $x \in \mathbb{R}^{n}$ and all $w \in F(x)$ we have $|w|_{\mathcal{A}} \leq \rho\left(|x|_{\mathcal{A}}\right)$ and

b) there exist $\alpha_{1} \in \mathcal{K}, \alpha_{2} \in \mathcal{G}$ such that for each $x_{0} \in \mathbb{R}^{n}$ and $\phi \in \mathcal{S}\left(x_{0}\right)$ we have

$$
\sum_{k=0}^{\infty} \alpha_{1}\left(\left|\phi\left(k, x_{0}\right)\right|_{\mathcal{A}}\right) \leq \alpha_{2}\left(\left|x_{0}\right|_{\mathcal{A}}\right) ;
$$

5) there exist $\alpha_{1} \in \mathcal{K}_{\infty}, \alpha_{2} \in \mathcal{G}$ such that for each $x_{0} \in \mathbb{R}^{n}$ and $\phi \in \mathcal{S}\left(x_{0}\right)$ inequality (12) holds;

6) a) $\mathcal{A}$ is UGS for system (1) and

b) for each pair of strictly positive real numbers satisfying $\delta \leq \Delta$ there exists a continuous function $\omega_{\delta, \Delta}: \mathbb{R}^{n} \rightarrow \mathbb{R}$ and strictly positive real numbers $\omega_{m}$ and $\gamma$ such that i) $\omega_{\delta, \Delta}(x) \geq \omega_{m}$ for all $x \in \mathcal{H}_{\mathcal{A}}(\delta, \Delta)$ and

ii) for all $x_{0} \in \mathcal{H}_{\mathcal{A}}(\delta, \Delta), \phi \in \mathcal{S}\left(x_{0}\right)$ and all $k \in \mathbb{N}$ we have

$$
\sum_{i=0}^{k} \omega_{\delta, \Delta}\left(\phi\left(i, x_{0}\right)\right) \leq \gamma
$$

Remark 5 A continuous-time counterpart of Theorem 2 is given in [15, Theorem 1] and its sampled-data counterpart can be found in [13].

\section{Detectability}

Let us consider now system (1) with an output $y \in \mathbb{R}^{p}$,

$$
y \in H(x),
$$

where $H(\cdot)$ is in general multi-valued. Given $x_{0} \in \mathbb{R}^{n}$, we denote by $\mathcal{S}_{H}\left(x_{0}\right)$ all possible pairs of trajectories and outputs that satisfy equations (1), (14), that is we write $(\phi, y) \in \mathcal{S}_{H}\left(x_{0}\right)$ if $\phi \in \mathcal{S}\left(x_{0}\right)$ and $y\left(j, x_{0}\right) \in H\left(\phi\left(j, x_{0}\right)\right)$ for all $j \in \mathbb{N}$.

Definition 6 Let $\alpha_{1}, \alpha_{2}, \alpha_{3} \in \mathcal{G}$. The closed set $\mathcal{A}$ is said to be $\left(\alpha_{1}, \alpha_{2}, \alpha_{3}\right)$-detectable for the system (1), (14) if for each $x_{0} \in \mathbb{R}^{n}$ and $(\phi, y) \in \mathcal{S}_{H}\left(x_{0}\right)$ we have

$$
\begin{aligned}
& \sum_{j=0}^{k} \alpha_{1}\left(\left|\phi\left(j, x_{0}\right)\right|_{\mathcal{A}}\right) \leq \alpha_{2}\left(\left|x_{0}\right|_{\mathcal{A}}\right)+\sum_{j=0}^{k} \alpha_{3}\left(\left|y\left(j, x_{0}\right)\right|\right) \\
& \forall k \in \mathbb{N} .
\end{aligned}
$$

The following statement follows as a corollary of previous theorems.

Corollary 1 Suppose that there exist $\alpha_{1} \in \mathcal{K}_{\infty}$ and $\alpha_{2}, \alpha, \bar{\alpha} \in \mathcal{G}$ such that the following conditions hold:

1) the closed set $\mathcal{A}$ is $\left(\alpha_{1}, \alpha_{2}, \alpha\right)$-detectable for the system (1), (14);

2) for each $x_{0} \in \mathbb{R}^{n}$ and $(\phi, y) \in \mathcal{S}_{H}\left(x_{0}\right)$ we have

$$
\sum_{k=0}^{\infty} \alpha\left(\left|y\left(k, x_{0}\right)\right|\right) \leq \bar{\alpha}\left(\left|x_{0}\right|_{\mathcal{A}}\right) \text {. }
$$

Then, the closed set $\mathcal{A}$ is UGAS for system (1). If moreover the pair $\left(\alpha_{1}, \alpha_{2}+\bar{\alpha}\right)$ is cUGES, the set $\mathcal{A}$ is UGES for system (1).

Proof. Combining (16) with $\left(\alpha_{1}, \alpha_{2}, \alpha\right)$-detectability, we have

$$
\sum_{k=0}^{\infty} \alpha_{1}\left(\left|\phi\left(k, x_{0}\right)\right|_{\mathcal{A}}\right) \leq \alpha_{2}\left(\left|x_{0}\right|_{\mathcal{A}}\right)+\bar{\alpha}\left(\left|x_{0}\right|_{\mathcal{A}}\right) .
$$

UGAS of $\mathcal{A}$ follows directly from Theorem 2 . If, moreover, the pair $\left(\alpha_{1}, \alpha_{2}+\bar{\alpha}\right)$ is cUGES, then UGES of $\mathcal{A}$ follows from Theorem 1.

\section{Remark 6}

- It is possible to modify the definition of detectability so that (15) holds with $\left|y\left(j, x_{0}\right)\right|_{\mathcal{B}}$ instead of $\left|y\left(j, x_{0}\right)\right|$ where $\mathcal{B} \subset \mathbb{R}^{p}$ is a closed set. With this modification, 
we would need to modify the condition (16) in the same manner.

- The condition in item 2 of the corollary holds e.g., if there exists a non-negative definite function $V$ and class $\mathcal{K}_{\infty}$ functions $\alpha_{1}$ and $\alpha_{2}$ such that $V(x) \leq$ $\alpha_{1}\left(|x|_{\mathcal{A}}\right)$ and $V(w) \leq V(x)-\alpha(|y|)$. Then, applying the sum from $k=0$ to infinity on both sides of the latter inequality, we recover (16). Hence, Corollary 1 establishes UGAS and UGES under detectability and "Krasovskii-LaSalle-type" conditions. Correspondingly, in [16] we present results which generalize the latter in the spirit of Matrosov's theorem $-c f$. [10], [9].

\section{PROOFS}

\section{A. Proof of Theorem 1}

$(1 \Longrightarrow 4)$ : Let item 1 and Definition 2 generate $\Gamma \in \mathbb{R}_{\geq 1}$ and $\lambda \in[0,1)$ such that (3) holds. Pick arbitrarily a cUGES pair $\left(\alpha_{1}^{-1}, \Gamma \alpha_{2}^{-1}\right)$ and, for any $\mu \in[0,1)$, let Lemma 2 generate $\Gamma_{*} \in \mathbb{R}_{\geq 1}$ and $\lambda_{*} \in[0,1)$ such that

$$
\mu^{k} \Gamma \alpha_{2}^{-1}(s) \leq \alpha_{1}^{-1}\left(s \Gamma_{*} \lambda_{*}^{k}\right)
$$

The latter holds, in particular, for $\mu=\lambda$ hence

$$
\alpha_{1}\left(\lambda^{k} \Gamma \alpha_{2}^{-1}(s)\right) \leq s \Gamma_{*} \lambda_{*}^{k} \quad \forall s \in \mathbb{R}_{\geq 0}, k \in \mathbb{N} .
$$

Therefore, for $s=\alpha_{2}\left(\left|x_{0}\right|_{\mathcal{A}}\right)$ and any $x_{0} \in \mathbb{R}^{n}$ we have, using (3),

$$
\alpha_{1}\left(\left|\phi\left(k, x_{0}\right)\right|_{\mathcal{A}}\right) \leq \alpha_{1}\left(\lambda^{k} \Gamma\left|x_{0}\right|_{\mathcal{A}}\right) \leq \alpha_{2}\left(\left|x_{0}\right|_{\mathcal{A}}\right) \Gamma_{*} \lambda_{*}^{k} .
$$

Evaluating the sum from $k=0$ to $\infty$ on both sides of the inequalities above, we obtain

$$
\sum_{k=0}^{\infty} \alpha_{1}\left(\left|\phi\left(k, x_{0}\right)\right|_{\mathcal{A}}\right) \leq \Gamma_{*} \sum_{k=0}^{\infty} \lambda_{*}^{k} \alpha_{2}\left(\left|x_{0}\right|_{\mathcal{A}}\right)
$$

so item 4 the theorem holds with $\Gamma_{2}:=\frac{\Gamma_{*}}{1-\lambda_{*}}$.

$(4 \Longrightarrow 3)$ : Let $\bar{\alpha}_{1}(s)=\bar{\alpha}_{2}(s)=s$. Then, $\left(\bar{\alpha}_{1}^{-1}, \Gamma \cdot \bar{\alpha}_{2}^{-1}\right)$ is cUGES for each $\Gamma \in \mathbb{R}_{\geq 1}$. Hence, using item 4 , there exists $\Gamma_{2} \in \mathbb{R}_{\geq 1}$ such that

$$
\sum_{k=1}^{\infty} \bar{\alpha}_{1}\left(\left|\phi\left(k, x_{0}\right)\right|_{\mathcal{A}}\right) \leq \Gamma_{2} \cdot \bar{\alpha}_{2}\left(\left|x_{0}\right|_{\mathcal{A}}\right) \text {. }
$$

Define $\alpha_{1}(s):=\bar{\alpha}_{1}(s)=s$ and $\alpha_{2}(s):=\Gamma_{2} \cdot \bar{\alpha}_{2}(s)=\Gamma_{2} \cdot s$ and note that the pair $\left(\alpha_{1}, \alpha_{2}\right)$ is cUGES. This and (20) immediately shows that item 3 of the theorem holds. $(3 \Longrightarrow 2)$ : By assumption we have

$$
\alpha_{1}\left(\left|\phi\left(1, x_{0}\right)\right|_{\mathcal{A}}\right) \leq \alpha_{2}\left(\left|x_{0}\right|_{\mathcal{A}}\right), \quad \forall x_{0} \in \mathbb{R}^{n}, \phi \in \mathcal{S}\left(x_{0}\right)
$$

or, equivalently, for all $x \in \mathbb{R}^{n}$ and $w \in F(x)$ we have

$$
|w|_{\mathcal{A}} \leq \alpha_{1}^{-1} \circ \alpha_{2}\left(|x|_{\mathcal{A}}\right)
$$

Since $\left(\alpha_{1}, \alpha_{2}\right)$ is cUGES by assumption, there exists $\Gamma_{1} \in$ $\mathbb{R}_{\geq 1}$ such that (5) holds.
We show next that system (1) is FSC. By assumption the pair $\left(\alpha_{1}, \alpha_{2}\right)$ is cUGES hence, by Lemma 2 there exist $\Gamma \in \mathbb{R}_{\geq 1}$ and $\lambda \in[0,1)$ such that for all $j \in \mathbb{N}$,

$$
0.5^{j} \alpha_{2}(s) \leq \alpha_{1}\left(\Gamma \lambda^{j} s\right) \quad \forall s \in \mathbb{R}_{\geq 0} .
$$

Let $j^{*} \in \mathbb{N}$ satisfy $\Gamma \lambda^{j^{*}} \leq 0.5$ and $k^{*} \in \mathbb{N}$ satisfy $\left(k^{*}+\right.$ $1)^{-1} \leq 0.5^{j^{*}}$. It follows from (23) that

$$
\left(k^{*}+1\right)^{-1} \alpha_{2}(s) \leq \alpha_{1}(0.5 s) \quad \forall s \in \mathbb{R}_{\geq 0}
$$

or equivalently,

$$
\alpha_{2}(s) \leq\left(k^{*}+1\right) \alpha_{1}(0.5 s) \quad \forall s \in \mathbb{R}_{\geq 0} .
$$

Next, we show by reductio ad absurdum that FSC holds with this $k^{*}$ and $\lambda_{\circ}=0.5$. Assume it does not i.e., suppose that there exists $x_{0} \in \mathbb{R}^{n}$ and $\phi \in \mathcal{S}\left(x_{0}\right)$ such that $\left|\phi\left(k, x_{0}\right)\right|_{\mathcal{A}}>0.5\left|x_{0}\right|_{\mathcal{A}}$ for all $k \in\left\{0,1, \ldots, k^{*}\right\}$. Then, since $\alpha_{1} \in \mathcal{K}_{\infty}$, we have

$\left(k^{*}+1\right) \alpha_{1}\left(0.5\left|x_{0}\right|_{\mathcal{A}}\right)<\sum_{k=0}^{k^{*}} \alpha_{1}\left(\left|\phi\left(k, x_{0}\right)\right|_{\mathcal{A}}\right) \leq \alpha_{2}\left(\left|x_{0}\right|_{\mathcal{A}}\right)$,

which contradicts (25).

$(2 \Longrightarrow 1)$ : Without loss of generality, assume that $\Gamma_{1} \in$ $\mathbb{R}_{\geq 1}$ and $\lambda_{\circ} \in(0,1)$. Then, from FSC we have that for any $x_{0}$ and $\phi \in \mathcal{S}\left(x_{0}\right)$ there exists a sequence of times $j_{i}, i \in \mathbb{Z}_{\geq 0}$, such that $j_{i+1}-j_{i} \leq k^{*}$ (and, consequently, $\left.j_{i} \leq i k^{*}\right)$ such that

$$
\left|\phi\left(j_{i}, x_{0}\right)\right|_{\mathcal{A}} \leq \lambda_{\circ}^{i}\left|x_{0}\right|_{\mathcal{A}} \leq\left(\lambda_{\circ}^{\frac{1}{k^{*}}}\right)^{j_{i}}\left|x_{0}\right|_{\mathcal{A}}=: \lambda^{j_{i}}\left|x_{0}\right|_{\mathcal{A}} .
$$

Moreover, from (5) we have that for any $k \in\left[j_{i}, j_{i+1}\right]$ the following holds:

$$
\begin{aligned}
\left|\phi\left(k, x_{0}\right)\right|_{\mathcal{A}} & \leq \Gamma_{1}^{k-j_{i}}\left|\phi\left(j_{i}, x_{0}\right)\right|_{\mathcal{A}} \\
& \leq \Gamma_{1}^{k-j_{i}} \lambda^{j_{i}}\left|x_{0}\right|_{\mathcal{A}} \\
& \leq \Gamma_{1}^{k^{*}} \lambda^{-\left(k-j_{i}\right)} \lambda^{k}\left|x_{0}\right|_{\mathcal{A}} \\
& \leq \Gamma_{1}^{k^{*}} \lambda^{-k^{*}} \lambda^{k}\left|x_{0}\right|_{\mathcal{A}} \\
& =\frac{\Gamma_{1}^{k^{*}}}{\lambda_{\circ}} \lambda^{k}\left|x_{0}\right|_{\mathcal{A}} .
\end{aligned}
$$

Hence, (3) holds with $\Gamma:=\Gamma_{1}^{k^{*}} / \lambda_{\circ}$ and $\lambda=\left(\lambda_{\circ}\right)^{1 / k^{*}}$.

\section{B. Proof of Theorem 2}

Without loss of generality we assume that functions $\rho \in$ $\mathcal{K}_{\infty}$ satisfy $\rho(s) \geq s, \forall s \geq 0$. Throughout this proof $\rho^{k}$ denotes the $k$-fold composition of the function $\rho$ with itself:

$$
\rho^{k}(s):=\underbrace{\rho \circ \rho \circ \cdots \circ \rho}_{k \text { times }}(s), \quad k \in \mathbb{Z}_{\geq 1} .
$$

Clearly, if $\rho \in \mathcal{K}_{\infty}$ then $\rho^{k} \in \mathcal{K}_{\infty}$ for each $k \in \mathbb{Z}_{\geq 1}$.

$(2 \Longrightarrow 1)$ :

Uniform stability: We first show that the origin is uni-

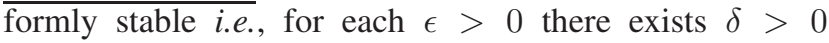
such that for all $x_{0} \in \mathbb{R}^{n}$ satisfying $\left|x_{0}\right|_{\mathcal{A}} \leq \delta$, we have $\left|\phi\left(k, x_{0}\right)\right|_{\mathcal{A}} \leq \epsilon$ for all $k \in \mathbb{N}$. Pick $\epsilon>0$ arbitrarily and 
let item 2(a) generate, via Definition 5, $k^{*}$ such that (9) holds for the pair $(r, \epsilon)=(\epsilon, \epsilon)$. Let $\delta_{0}>0$ be such that $\rho^{k^{*}}\left(\delta_{0}\right) \leq \epsilon$ and define $\delta=\min \left\{\epsilon, \delta_{0}\right\}$. Using this and item 2(b) it is now straightforward to verify that $\left|x_{0}\right|_{\mathcal{A}} \leq \delta$ implies $\left|\phi\left(k, x_{0}\right)\right|_{\mathcal{A}} \leq \epsilon$ for all $k \in \mathbb{N}$.

Uniform global boundedness: Next we establish uniform global boundedness i.e., there exist $\mu \geq 0$ and $\gamma \in \mathcal{K}_{\infty}$ such that, for all initial conditions and all solutions we have $\left|\phi\left(k, x_{0}\right)\right|_{\mathcal{A}} \leq \mu+\gamma\left(\left|x_{0}\right|_{\mathcal{A}}\right)$. We take $\mu=1$. Let $\delta>0$ come from uniform stability for $\epsilon=1$. Then, for each $\Delta \geq \delta$, let $k^{*}(\Delta)$ come from uniform global attractivity for $(r, \epsilon)=(\Delta, \Delta)$. Without loss of generality, we can assume that $k^{*}(\cdot)$ is non decreasing on $[\delta, \infty)$. Then, it may be verified that for all $x_{0} \in \mathbb{R}^{n}$ satisfying $\left|x_{0}\right|_{\mathcal{A}} \geq \delta$, we have

$$
\left|\phi\left(k, x_{0}\right)\right|_{\mathcal{A}} \leq \rho^{k^{*}\left(\left|x_{0}\right|_{\mathcal{A}}\right)}\left(\left|x_{0}\right|_{\mathcal{A}}\right) \quad \forall k \in \mathbb{N} .
$$

Finally, we let $\gamma$ be any function in class- $K_{\infty}$ satisfying $\rho^{k^{*}(s)}(s) \leq \gamma(s)$ for all $s \in[\delta, \infty)$. It now can be verified that for all initial conditions, solutions and $k \in \mathbb{N}$, we have $\left|\phi\left(k, x_{0}\right)\right|_{\mathcal{A}} \leq \mu+\gamma\left(\left|x_{0}\right|_{\mathcal{A}}\right)$.

Uniform stability and uniform global boundedness imply uniform global stability:

This is seen as follows: We take the uniform stability relationship $\epsilon \mapsto \delta(\epsilon)>0$ and find a class- $\mathcal{K}$ function $\eta$ such that $\eta(\epsilon) \leq \delta(\epsilon)$ for all $\epsilon>0$. Next we note that $\eta$ can be inverted on its range, denoted $\left[0, \eta_{\infty}\right)$. If $\eta_{\infty}=\infty$ then we define $\rho_{2}:=\eta^{-1}$. Otherwise let $\eta^{*} \in\left(0, \eta_{\infty}\right)$ satisfy $\eta^{-1}\left(\eta^{*}\right)=\mu+\gamma\left(\eta^{*}\right)$ and define

$$
\rho_{2}(s):=\left\{\begin{array}{cc}
\eta^{-1}(s) & s \in\left[0, \eta^{*}\right] \\
\mu+\gamma(s) & s \geq \eta^{*}
\end{array}\right.
$$

It is straightforward to see that $\rho_{2} \in \mathcal{K}_{\infty}$ and that the uniform global stability bound holds with $\rho_{2}$.

Uniform global stability and uniform global attractivity imply UGAS:

Regarding the mapping $(\epsilon, \Delta) \mapsto k^{*}(\epsilon, \Delta)$ that comes from uniform global attractivity, we can assume without loss of generality that

- for each $\Delta>0, k^{*}(\cdot, \Delta)$ is non-increasing on $\mathbb{R}_{>0}$ and, with uniform global stability, $k^{*}(\epsilon, \Delta)=0$ for $\epsilon$ sufficiently large i.e., $\epsilon \geq \rho_{2}(\Delta)$;

- for each $\epsilon>0 k^{*}(\epsilon, \cdot)$ is nondecreasing on $\mathbb{R}_{>0}$.

Let $\psi_{\Delta}: \mathbb{R}_{>0} \rightarrow \mathbb{R}_{>0}$ be a function that is strictly decreasing and onto $\mathbb{R}_{>0}$ (hence invertible on $\mathbb{R}_{>0}$ ) and satisfies

$$
\psi_{\Delta}(\epsilon) \geq k^{*}(\Delta, \epsilon) \quad \forall \epsilon>0 .
$$

We claim that $\left|x_{0}\right|_{\mathcal{A}} \leq \Delta$ implies $\left|\phi\left(k, x_{0}\right)\right|_{\mathcal{A}} \leq \psi_{\Delta}^{-1}(k)$ for all $k \in \mathbb{Z}_{\geq 1}$. To see this, for each $k \in \mathbb{Z}_{\geq 1}$ let $\epsilon:=\psi_{\Delta}^{-1}(k)$ and then note that, from (29), $\psi_{\Delta}(\epsilon)=$ $k \geq k^{*}(\Delta, \epsilon)$. Therefore, from uniform global attractivity, $\left|\phi\left(k, x_{0}\right)\right|_{\mathcal{A}} \leq \epsilon=\psi_{\Delta}^{-1}(k)$. Finally, for each $\Delta>0$ define $\psi_{\Delta}^{-1}(0):=\infty$ and define

$$
\beta(s, k):=\min \left\{\rho_{2}(s), \inf _{\Delta \in(s, \infty)} \psi_{\Delta}^{-1}(k)\right\} .
$$

It is straightforward to verify that $\beta \in \mathcal{K} \mathcal{L}$ and that $\left|\phi\left(k, x_{0}\right)\right|_{\mathcal{A}} \leq \beta\left(\left|x_{0}\right|_{\mathcal{A}}, k\right)$ for all $x_{0} \in \mathbb{R}^{n}$ and $k \in \mathbb{N}$.

$(3 \Longrightarrow 2)$ : We only need to establish uniform global attractivity. Let $\Delta>0$ and $\epsilon>0$ be given. Using the function $\rho \in \mathcal{K}_{\infty}$ from uniform global stability, let $\delta:=\rho^{-1}(\epsilon)$. Then $\left|x_{0}\right|_{\mathcal{A}} \leq \delta$ implies $\left|\phi\left(k, x_{0}\right)\right|_{\mathcal{A}} \leq \epsilon$ for all $k \in \mathbb{N}$. Define

$$
\eta^{*}=\min _{s \in[\delta, \rho(\Delta)]} \eta(s) .
$$

Then let $k^{*}$ be the smallest nonnegative integer satisfying

$$
k^{*} \geq \frac{\alpha_{2}(\Delta)}{\eta^{*}}-1 .
$$

We claim that for each $x_{0}$ satisfying $\left|x_{0}\right|_{\mathcal{A}} \leq \Delta$ and $\phi \in \mathcal{S}\left(x_{0}\right)$, there exists $\bar{k} \in\left\{0,1, \ldots, k^{*}\right\}$ such that $\left|\phi\left(\bar{k}, x_{0}\right)\right|_{\mathcal{A}} \leq \delta$. If not then

$$
\begin{aligned}
\sum_{k=0}^{\infty} \eta\left(\left|\phi\left(k, x_{0}\right)\right|_{\mathcal{A}}\right) \geq & \sum_{k=0}^{k^{*}} \eta\left(\left|\phi\left(k, x_{0}\right)\right|_{\mathcal{A}}\right) \\
& >\left(k^{*}+1\right) \eta^{*} \\
& \geq \alpha_{2}(\Delta) \geq \alpha_{2}\left(\left|x_{0}\right|_{\mathcal{A}}\right) .
\end{aligned}
$$

Considering $\phi\left(k, x_{0}\right)$ for $k \geq \bar{k}$ as a solution starting at $\phi\left(\bar{k}, x_{0}\right)$, it follows that $\left|x_{0}\right|_{\mathcal{A}} \leq \Delta$ and $k \geq k^{*}$ imply $\left|\phi\left(k, x_{0}\right)\right|_{\mathcal{A}} \leq \epsilon$.

( $4 \Longrightarrow 2$ ): This implication is very similar to the previous one. We just need to establish uniform global attractivity. Let $\Delta>0$ and $\epsilon>0$ be given. Let $\delta>0$ be such that $\alpha_{1}(s) \leq \alpha_{2}(\delta)$ implies $s \leq \epsilon$. Such a $\delta$ exists since $\alpha_{1} \in \mathcal{K}$ and $\alpha_{2} \in \mathcal{G}$. Note that $\left|x_{0}\right|_{\mathcal{A}} \leq \delta$ implies $\left|\phi\left(k, x_{0}\right)\right|_{\mathcal{A}} \leq \epsilon$ for all $k \in \mathbb{N}$ since

$$
\begin{aligned}
& \alpha_{1}\left(\left|\phi\left(k, x_{0}\right)\right|_{\mathcal{A}}\right) \leq \sum_{i=0}^{\infty} \alpha_{1}\left(\left|\phi\left(i, x_{0}\right)\right|_{\mathcal{A}}\right) \\
& \leq \alpha_{2}\left(\left|x_{0}\right|_{\mathcal{A}}\right) \leq \alpha_{2}(\delta) .
\end{aligned}
$$

Next let $k^{*}$ be the smallest nonnegative integer satisfying

$$
k^{*} \geq \frac{\alpha_{2}(\Delta)}{\alpha_{1}(\delta)}-1 .
$$

We claim that for each $x_{0}$ satisfying $\left|x_{0}\right|_{\mathcal{A}} \leq \Delta$ and $\phi \in \mathcal{S}\left(x_{0}\right)$, there exists $\bar{k} \in\left\{0, \ldots, k^{*}\right\}$ such that $\left|\phi\left(\bar{k}, x_{0}\right)\right|_{\mathcal{A}} \leq \delta$. If not then

$$
\begin{aligned}
\sum_{k=0}^{\infty} \alpha_{1}\left(\left|\phi\left(k, x_{0}\right)\right|_{\mathcal{A}}\right) \geq & \sum_{k=0}^{k^{*}} \alpha_{1}\left(\left|\phi\left(k, x_{0}\right)\right|_{\mathcal{A}}\right) \\
& >\left(k^{*}+1\right) \alpha_{1}(\delta) \\
& \geq \alpha_{2}(\Delta) \geq \alpha_{2}\left(\left|x_{0}\right|_{\mathcal{A}}\right) .
\end{aligned}
$$

Considering $\phi\left(k, x_{0}\right)$ for $k \geq \bar{k}$ as a solution starting at $\phi\left(\bar{k}, x_{0}\right)$, it follows that $\left|x_{0}\right| \leq \Delta$ and $k \geq k^{*}$ imply $\left|\phi\left(k, x_{0}\right)\right|_{\mathcal{A}} \leq \epsilon$.

( $5 \Longrightarrow 4$ ): We only need to establish part (a) of item 4 since part (b) is obvious. By assumption $\alpha_{1}\left(|w|_{\mathcal{A}}\right) \leq \alpha_{2}\left(\left|x_{0}\right|_{\mathcal{A}}\right)$ for all $x_{0} \in \mathbb{R}^{n}$ and $w \in F\left(x_{0}\right)$. Thus $|w|_{\mathcal{A}} \leq \alpha_{1}^{-1} \circ$ $\alpha_{2}\left(\left|x_{0}\right|_{\mathcal{A}}\right)=: \rho\left(\left|x_{0}\right|_{\mathcal{A}}\right)$. This establishes the result. 
( $5 \Longrightarrow 3$ ): We only need to establish part (a) of item 3 since part (b) is obvious. By assumption, for all $k \in \mathbb{N}$, all $x_{0} \in \mathbb{R}^{n}$

$$
\alpha_{1}\left(\left|\phi\left(k, x_{0}\right)\right|_{\mathcal{A}}\right) \leq \sum_{i=0}^{\infty} \alpha_{1}\left(\left|\phi\left(i, x_{0}\right)\right|_{\mathcal{A}}\right) \leq \alpha_{2}\left(\left|x_{0}\right|_{\mathcal{A}}\right)
$$

or, equivalently, $\left|\phi\left(k, x_{0}\right)\right|_{\mathcal{A}} \leq \alpha_{1}^{-1} \circ \alpha_{2}\left(\left|x_{0}\right|_{\mathcal{A}}\right)$ i.e., the origin is uniformly globally stable.

(1 $\Longrightarrow 5$ ): According to Lemma 1 , there exists $\alpha_{1}, \alpha_{2} \in$ $\mathcal{K}_{\infty}$ such that

$$
\alpha_{1}(\beta(s, k)) \leq \alpha_{2}(s)\left(\frac{1}{2}\right)^{k+1} \quad \forall(s, k) \in \mathbb{R}_{\geq 0} \times \mathbb{N} .
$$

Then

$\sum_{k=0}^{\infty} \alpha_{1}\left(\left|\phi\left(i, x_{0}\right)\right|_{\mathcal{A}}\right) \leq \sum_{k=0}^{\infty} \alpha_{2}\left(\left|x_{0}\right|_{\mathcal{A}}\right)\left(\frac{1}{2}\right)^{k+1}=\alpha_{2}\left(\left|x_{0}\right|_{\mathcal{A}}\right)$

(3 $\Longrightarrow$ ): Since items 3(a) and 6(a) are identical, we only need to show that $3(\mathrm{~b})$ implies $6(\mathrm{~b})$. This is immediate with the following definitions $\omega_{\delta, \Delta}(x):=\eta\left(|x|_{\mathcal{A}}\right), \omega_{m}:=$ $\min _{s \in[\delta, \Delta]} \eta(s), \gamma:=\alpha_{2}(\Delta)$. Then, item 6(b)i holds by definition of $\omega_{m}$ and 6(b)ii holds because for all $x_{0} \in$ $\mathcal{H}_{\mathcal{A}}(\delta, \Delta), \phi \in \mathcal{S}\left(x_{0}\right)$ and all $k \in \mathbb{N}$ we have

$$
\begin{aligned}
\sum_{i=0}^{k} \omega_{\delta, \Delta}\left(\phi\left(i, x_{0}\right)\right) \leq & \sum_{i=0}^{\infty} \eta\left(\left|\phi\left(i, x_{0}\right)\right|_{\mathcal{A}}\right) \\
& \leq \alpha_{2}\left(\left|x_{0}\right|_{\mathcal{A}}\right) \leq \alpha_{2}(\Delta)=\gamma
\end{aligned}
$$

(6 $\Longrightarrow 1$ ): Since $\mathcal{A}$ is assumed to be UGS we only need to prove UGA. From item $6(\mathrm{a})$, let $\rho \in \mathcal{K}_{\infty}$ be such that for all $x_{0} \in \mathbb{R}^{n}, \phi \in \mathcal{S}\left(x_{0}\right)$ and $k \in \mathbb{N}$ we have

$$
|\phi(k, x)|_{\mathcal{A}} \leq \rho\left(\left|x_{0}\right|_{\mathcal{A}}\right) \text {. }
$$

Let the strictly positive numbers $(r, \epsilon)$ be given. Define $\Delta:=\rho(r)$ and $\delta:=\min \left\{\Delta, \rho^{-1}(\epsilon)\right\}$. Let $\delta, \Delta$ generate $\omega_{\delta, \Delta}(x), \omega_{m}$ and $\gamma$. Define $k^{*}:=\max \left\{j \in \mathbb{N}: j \leq \frac{2 \gamma}{\omega_{m}}\right\}$. We claim that for all $x_{0} \in \mathcal{H}_{\mathcal{A}}(0, r)$ and all $\phi \in \mathcal{S}\left(x_{0}\right)$ there exists $k^{\prime} \in\left[0, k^{*}\right]$ such that $\left|\phi\left(k^{\prime}, x_{0}\right)\right|_{\mathcal{A}} \leq \rho^{-1}(\epsilon)$. This establishes the result since the time invariance of the system and (37) imply that for all $x_{0} \in \mathcal{H}_{\mathcal{A}}(0, r)$ and $k \geq k^{*}$ we have $\left|\phi\left(k, x_{0}\right)\right|_{\mathcal{A}} \leq \epsilon$.

Assume that the claim is not true that is, there exists $x_{0} \in \mathcal{H}_{\mathcal{A}}(0, r)$ and $\phi \in \mathcal{S}\left(x_{0}\right)$ such that $\left|\phi\left(k, x_{0}\right)\right|_{\mathcal{A}}>$ $\rho^{-1}(\epsilon)$ for all $k \in\left[0, k^{*}\right]$. From (37) and the definition of $\delta$ it follows that

$$
\phi\left(k, x_{0}\right) \in \mathcal{H}_{\mathcal{A}}(\delta, \Delta) \quad \forall k \in\left[0, k^{*}\right] .
$$

It follows that $\omega_{\delta, \Delta}\left(\phi\left(k, x_{0}\right)\right) \geq \omega_{m}$ for all $k \in\left[0, k^{*}\right]$. Hence,

$$
\sum_{i=0}^{k^{*}} \omega_{\delta, \Delta}\left(\phi\left(i, x_{0}\right)\right) \geq\left(k^{*}+1\right) \omega_{m} \geq \frac{2 \gamma}{\omega_{m}} \omega_{m}=2 \gamma,
$$

which contradicts $6(\mathrm{~b})$. Hence, the set $\mathcal{A}$ is UGA for system (1).

\section{COnClusions}

We have provided several results that can be used to verify UGAS and UGES of arbitrary closed sets that do not require the knowledge of Lyapunov functions. Instead, we assume appropriate summability conditions on trajectories of the system. The results and their proofs presented here parallel the continuous-time and sampled-data counterparts but they are more straightforward and derived under different assumptions.

\section{REFERENCES}

[1] E. A. Barbashin and N. N. Krasovskiĭ. O cyschestvovanii functziǐ Lyapunova v cluchae asimptoticheskoŭ ustořchivosť v tzelom. Prikl. Mat. i Mekh., 18:345-350, 1954. English title: "On the existence of Lyapunov fuctions in the case of asymptotic stability in the large". English apropriate translation: On the existence of Lyapunov fuctions in the case of asymptotic stability in the whole.

[2] P. Giesel. Necessary conditions for the basin of attraction of a periodic orbit of non-smooth periodic systems. Discrete and Continuous Dynamical Systems, 18(2\& 3):355-373, 2007.

[3] Rafal Goebel, Tingshu Hu, and Andrew R. Teel. Dual matrix inequalities in stability and performance analysis of linear differential/difference inclusions. In Current trends in nonlinear systems and control, Systems Control Found. Appl., pages 103-122. Birkhäuser Boston, Boston, MA, 2006.

[4] G. Grammel. Towards fully discretized differential inclusions. Setvalued Analysis, 11:1-8, 2003.

[5] W. Hahn. Theory and Application of Liapunov's Direct Method. Prentice Hall, New York, 1963.

[6] S. Hui and S. Zak. On the Lyapunov stability of discrete-time processes modeled by difference inclusions. Syst. \& Contr. Letters, 10(3):207-299, 1988.

[7] P. E. Kloeden and B. Schmalfuss. Lyapunov functions and attractors under variable time-step discretization. Discrete and Continuous Dynamical Systems, 2(2):163-172, 1996.

[8] A. Loría F. Lamnabhi-Lagarrigue, and D. Nesic. Summationtype conditions for uniform asymptotic convergence in discrete-time systems: applications in identification. In Proc. 44th. IEEE Conf. Decision Contr., pages 6591-6595, 2005.

[9] A. Loría E. Panteley, D. Popovic, and A. Teel. A nested Matrosov theorem and persistency of excitation for uniform convergence in stable non-autonomous systems. IEEE Trans. on Automat. Contr. 50(2):183-198, 2005.

[10] V. M. Matrosov. On the stability of motion. J. Appl. Math. Mech., 26:1337-1353, 1962.

[11] D. Nešić and A. Teel. Matrosov theorem for parameterized families of discrete-time systems. Automatica, 40(6):1025-1034, 2004.

[12] D. Nešić and A. R. Teel. A framework for stabilization of nonlinear sampled-data systems based on their approximate discrete-time models. IEEE Trans. on Automat. Contr., 49(7):1103-1122, 2004.

[13] D. Nešić, A. Loría, E. Panteley, and A. R. Teel. On stability of sets for sampled-data nonlinear inclusions via their approximate discretetime models and summability criteria. SIAM Journal on Control and Optimization, 48(3):1888-1913, 2009.

[14] E. D. Sontag. Comments on integral variants of ISS. Syst. \& Contr. Letters, 34:93-100, 1998.

[15] A. Teel, E. Panteley, and A. Loría. Integral characterizations of uniform asymptotic and exponential stability with applications. Math. of Cont. Sign. and Syst., 15:177-201, 2002.

[16] A. R. Teel, D. Nešić, A. Loría and E. Panteley. Summability criteria for uniform exponential and asymptotic set-stability of difference inclusions. Journal of Difference Equations and Applications, 2009 In Press.

[17] S. K. Zavriyev and A. G. Perevozchikov. The direct Lyapunov method in investigating the attraction of trajectories of finitedifference inclusions. USSR Computational Mathematics and Mathematical Physics, 30(1):15-22, 1990. 